\title{
Hedgehog (Hh) Signaling is a Predictor of Clinical Outcome for Advanced Non- Small Cell Lung Cancer (NSCLC)
}

Berardi $\mathbf{R}^{1^{*}}$, Santinelli $\mathbf{A}^{2}$, Onofri $\mathbf{A}^{1}$, Biscotti $\mathbf{T}^{2}$, Ballatore $\mathbf{Z}^{1}$, Caramanti $\mathbf{M}^{1}$, Savini $\mathbf{A}^{1}$, De Lisa $\mathbf{M}^{1}$, Morgese $\mathbf{F}^{1}$, Pompili $\mathbf{C}^{3}$, Salati $\mathbf{M}^{3}$, Chiorrini $\mathbf{S}^{1}$, Brunelli $\mathbf{A}^{3}$, Mazzanti $\mathbf{P}^{1}$, Bearzi $\mathbf{I}^{2}$ and Cascinu $\mathbf{S}^{1}$

${ }^{1}$ Clinica di Oncologia Medica, Università Politecnica delle Marche, AOU Ospedali Riuniti di Ancona, Italy

${ }^{2}$ Anatomia Patologica, Università Politecnica delle Marche, AOU Ospedali Riuniti di Ancona, Italy

${ }^{3}$ Chirurgia Toracica, AOU Ospedali Riuniti di Ancona, Italy

${ }^{4}$ Department of Thoracic Surgery, St. James's University Hospital, Leeds, UK

*Corresponding author: Rossana Berardi, MD, Medical Oncology Unit, Università Politecnica delle Marche - Azienda Ospedaliero-Universitaria, Ospedali Riuniti Umberto I - GM Lancisi - G Salesi di Ancona, Via Conca 71 - 60126 Ancona - Italy, Tel: +39 071 5965715; Fax: +39 071 5965053; E-mail: r.berardi@univpm.it

Recieved date: Feb 21, 2014, Accepted date: May 23, 2014, Pulbished date:May 29, 2014

Copyright: ( 2014 Berardi R, et al. This is an open-access article distributed under the terms of the Creative Commons Attribution License, which permits unrestricted use, distribution, and reproduction in any medium, provided the original author and source are credited.

\section{Abstract}

Objective: Lung cancer still remains the leading cause of cancer deaths in the world and it lacks validated biomarkers to predict clinical outcome. The Hedgehog $(\mathrm{Hh})$ pathway is critical for cell growth and differentiation. The aim of our study was to evaluate the role of the $\mathrm{Hh}$ signaling in the prediction of clinical outcome for advanced NSCLC.

Methods: We determined the expression of Hh-related molecules including Ptch1 and Gli1 (nuclear and cytoplasmic) by immunohistochemistry in histologic samples (biopsies and surgical specimens) of advanced NSCLC patients. All the neoplastic area included in the slides was considered and both cytoplasmic and nuclear stainings were evaluated, according to the positive neoplastic cells. The intensity of the staining was evaluated and scored as follows: 0 (absent), 1+ (low), 2+ (medium) and 3+ (high).

Results: We analyzed 35 lung cancer histological samples, 18 adenocarcinomas and 17 squamous cell carcinomas. Positivity of Gli1-cytoplasmic and Gli1-nuclear expression was expressed in adenocarcinoma at a significantly higher level and more frequently than compared to squamous cell carcinoma $(p<0.05)$, while Ptch1 did not differ significantly in the two histotypes. Overall survival was longer in Gli1 and Ptch1 negative tumor samples compared to the positive group $(p=0.02)$. The 18 patients with adenocarcinoma received erlotinib as second line therapy and those presenting a lower Gli1 and Ptch1 expression experienced a significant increase in progression free survival.

Conclusion: At our best knowledge this represents the first study investigating the role of HH in NSCLC patients. Our results suggest that the Hh pathway might play a major prognostic role in NSCLC with significant differences between the histotypes.

Keywords: Hedgehog signaling; Ptch1; Gli; Non-small cell lung cancer; Different hystoype; Prognosis; Predictive factor

\section{Introduction}

Despite the recent progresses in the diagnosis and treatment, nonsmall cell lung cancer (NSCLC) remains the leading cause of death due to cancer worldwide [1-3].

Increasing knowledge and recent progresses in molecular biology lead up to find many biological and molecular markers that may represent prognostic factors and may impact on the choice of new therapeutic strategies.

Although not completely established, it is suggested that Hedgehog (Hh) signaling seems to play an important role for cancer stem cells in several cancer type [4]. Constitutive activation of the Hh/GLI signaling cascade has recently been implicated in the growth of a number of human malignancies ranging from semi-malignant tumors of the skin to highly aggressive cancers of the brain, lung, pancreas and prostate
[5]. Furthermore, Hh has recently been recognized to be one of the most important signaling pathways and a therapeutic target [6]. In view of the critical role of GLI genes in Hh-associated cancers, Strategies that aim at interfering with GLI function are likely to represent efficient approaches in future targeted cancer therapy [5].

The Hh signaling cascade is initiated by the Hh ligand binding to the Patched 1 protein (PTCH1), a 12-span transmembrane protein. In the absence of a Hh ligand, PTCH1 catalytically inhibits the activity of the seven-transmembrane-span receptor-like protein, Smoothened (SMO), potentially by affecting its localization to the cell surface $[7,8]$.

Hh ligands bind to PTCH1, causing internalization and degradation, thereby releasing SMO that interacts with the protein Suppressor of fused (SUFU), which promotes the activation and nuclear translocation of glioma-associated oncogene homologue 1 and 2 (GLI1 GLI2) and the degradation of GLI3. Activated GLI proteins stimulate the transcription of Hh pathway target genes, including GLI1, GLI2 and PTCH1 [9] (Figure 1). 


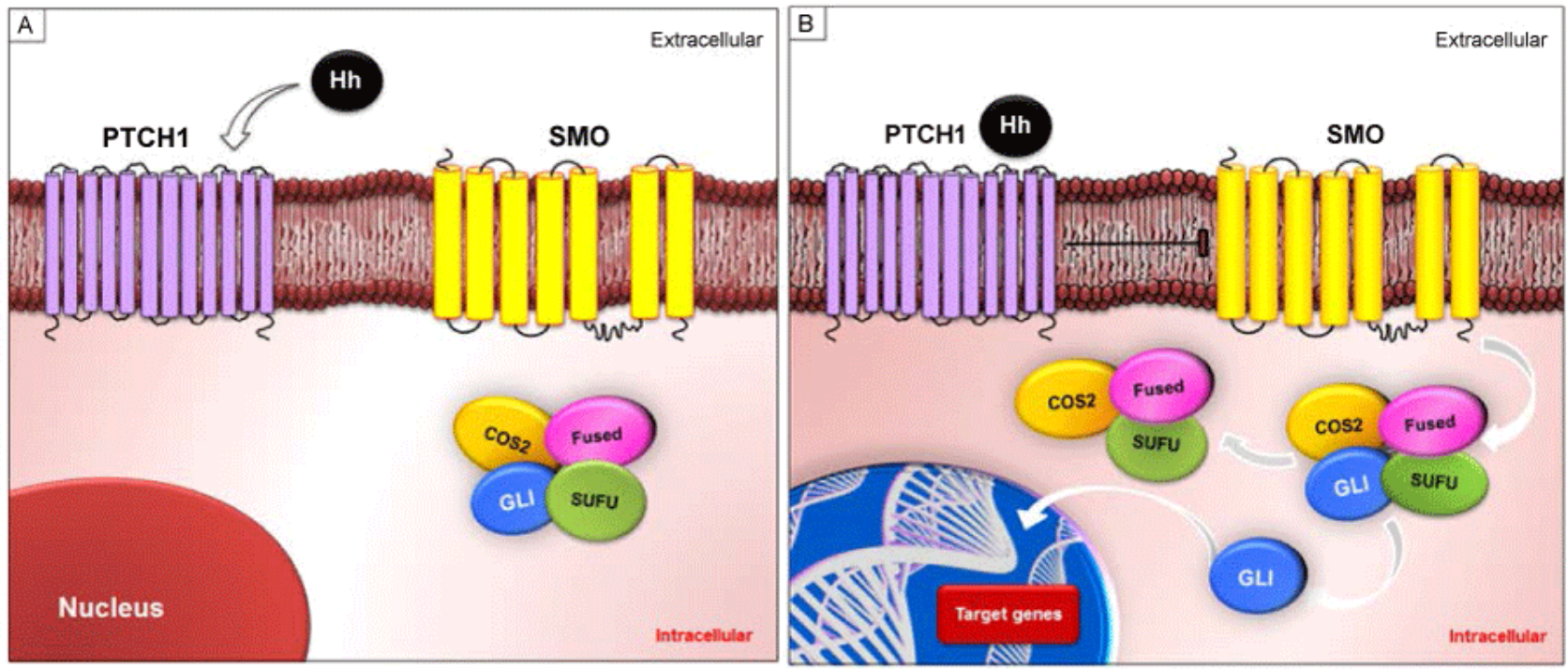

Figure 1: Hedgehog pathway: h ligands ( $\mathrm{SHH}, \mathrm{DHH}$ or $\mathrm{IHH}$ ) bind to the PTCH receptors (PTCH1 and PTCH2) and relieve the inhibition of SMO. SMO then transduces signals through the cytoplasmic SUFU-GLI complex, resulting in the activation and nuclear translocation of the downstream GLI transcription factors (GLI1-3).

Mutation or deregulation of this pathway plays a key role in both proliferation and differentiation leading to tumorigenesis or tumor growth acceleration in a wide variety of tissues $[10,11]$.

In particular, $\mathrm{Hh}$ signaling pathological activation has been reported both in small-cell-lung cancer (SCLC) and NSCLC [12-15]. Recently, a subset of NSCLC was found to be constitutively hyperactive in the Hh/GLI pathway by expressing high level of GLI1 protein [12] which was expressed in most SCLC tumors studied too [13].

Nevertheless, just one study investigated the potential role of $\mathrm{Hh}$ on the outcome of advanced NSCLC [16].

Several studies are ongoing to evaluate the potential role of $\mathrm{Hh}$ inhibitors as targeted therapies in solid tumors, therefore we investigated the contribution of Hh pathway to NSCLC progression and clinical outcome. Furthermore, we also evaluate the outcome in NSCLC patients treated with erlotinib.

\section{Patients and Methods}

\section{Patients selection}

This study includes consecutive patients with advanced NSCLC treated at the Department of Medical Oncology Università Politecnica Marche in Italy between January 2009 and December 2011.

Recorded patient characteristics and clinical features included the following: age, sex, smoking history, Eastern Cooperative Oncology Group (ECOG) performance status, histological type, clinical and/or pathological stage of disease (T, N, M) according to the TNM Seventh Edition (2010), data regarding all the treatments performed by the patients $[17,18]$.

Response to therapy was assessed according to the RECIST 1.1 (Response Evaluation Criteria In Solid Tumors) [19].

\section{Pathologic analysis}

In 35 histological samples of consecutive NSCLC patients the expression of Hh-related molecules, including Patched (G-19) and Gli1 (H-300), was determined by immunohistochemistry.

Immunohistochemical studies were carried out with the Envision method, consisting of a polymer of dextran to which many molecules both of secondary antibody and enzymes are linked.

Sections of 3-5 $\mu \mathrm{m}$ thick were deparaffinized and the detection of antigens has occurred in automated manner with DAKO PT Link using ENVISION ${ }^{\mathrm{m}}$ FLEX TARGET RETRIEVAL SOLUTION LOW $\mathrm{pH}(50 \mathrm{X})(\mathrm{DAKO})$ at a temperature of $98^{\circ} \mathrm{C}$.

After 70 minutes of treatment, sections were treated with $3 \%$ hydrogen peroxide and incubated at $4^{\circ} \mathrm{C}$ overnight with primary polyclonal antibodies against Patched (G-19) (1:25, Santa Cruz Biotechnology, Santa Cruz, CA) and Gli1 (H-300) (1:25, Santa Cruz Biotechnology, Santa Cruz, CA). The staining was completed using ENVISION FLEX "m/HRP (DAKO), as detection system; 3,3diaminobenzidine-hydrogen peroxide was used as chromogen. Human liver was used as a positive control for Glil and human testis was used as a positive control for Patched.

Protein Gli1 (H-300) expression was evaluated both in the cytoplasm and in the nucleus of neoplastic cells, while the expression of Patched (G-19) was evaluated only in the cytoplasm. Protein Gli1 (H-300) expression was evaluated both in the cytoplasm and in the nucleus of neoplastic cells, while the expression of Patched (G-19) was evaluated only in the cytoplasm. Smo (N-19) and Shh (N-19) (Santa Cruz Biotechnology) were tested immunoistochemically but we did not obtain reliable and reproducible results. For this reason we decided to not include them in our study.

\begin{tabular}{|l|l|}
\hline Patients Data & N (\%)
\end{tabular}


Citation: Berardi R, Santinelli A, Onofri A, Biscotti T, Ballatore Z, et al. (2014) Hedgehog (Hh) Signaling is a Predictor of Clinical Outcome for Advanced Non-Small Cell Lung Cancer (NSCLC). J Carcinog Mutagen 5: 175. doi:10.4172/2157-2518.1000175

Page 3 of 7

\begin{tabular}{|c|c|}
\hline Number of patients & 35 \\
\hline Median age [range], years & $71[40-82]$ \\
\hline Male/Female & $29 / 6(82.9 / 17.1)$ \\
\hline \multicolumn{2}{|l|}{ Smoking status } \\
\hline Smoker & $25(71,4)$ \\
\hline No Smoker & $8(22.9)$ \\
\hline Unknown & $2(5.7)$ \\
\hline \multicolumn{2}{|c|}{ Performance Status at the onset (ECOG) } \\
\hline 0 & $20(57.1)$ \\
\hline 1 & $14(40)$ \\
\hline 2 & $1(2.9)$ \\
\hline \multicolumn{2}{|l|}{ Stage at initial diagnosis } \\
\hline । & $2(5.7)$ \\
\hline IIA & $2(5.7)$ \\
\hline IIB & $1(2.9)$ \\
\hline III-IV & $30(85.7)$ \\
\hline \multicolumn{2}{|c|}{ Sites of metastases at the onset } \\
\hline Bone & 8 \\
\hline Lung & 8 \\
\hline Adrenal gland & 3 \\
\hline Lymph nodes & 3 \\
\hline Pleura & 3 \\
\hline Brain & 2 \\
\hline Liver & 1 \\
\hline Other & 0 \\
\hline \multicolumn{2}{|l|}{ Death } \\
\hline Yes & $12(34.3)$ \\
\hline No & $23(65.7)$ \\
\hline \multicolumn{2}{|l|}{ Histotype } \\
\hline Squamous cell carcinoma & $17(48.6)$ \\
\hline Adenocarcinoma & $18(51.4)$ \\
\hline \multicolumn{2}{|l|}{ Surgery } \\
\hline Not performed & $20(57.1)$ \\
\hline Performed & $15(42.9)$ \\
\hline Lobectomy & $10 / 15(66.7)$ \\
\hline Segmentectomy & $1 / 15(6.7)$ \\
\hline Pneumonectomy & $3 / 15(20)$ \\
\hline Other & $1 / 15(6.7)$ \\
\hline
\end{tabular}

\begin{tabular}{|c|c|}
\hline \multicolumn{2}{|l|}{ Radical surgery } \\
\hline Yes & $14 / 15$ \\
\hline No & $1 / 15(6.7)$ \\
\hline \multicolumn{2}{|c|}{ Adjuvant therapy } \\
\hline Not performed & 7/15 (46.7) \\
\hline Performed & $7 / 15(46.7)$ \\
\hline Unknown & $1 / 15(6.7)$ \\
\hline $\mathrm{Ct}$ & $5 / 7(71.4)$ \\
\hline Rt & 1/7 (14.3) \\
\hline $\mathrm{Ct}+\mathrm{rt}$ & 1/7 (14.3) \\
\hline \multicolumn{2}{|l|}{ Radiotherapy } \\
\hline Not performed & $21 / 35(60)$ \\
\hline Performed & $14 / 35(40)$ \\
\hline
\end{tabular}

Table 1: Clinical and pathological characteristics of patients and Treatments received. $\mathrm{CT}=$ chemotherapy, $\mathrm{RT}=$ radiotherapy.

Cytoplasmic and nuclear immunohistochemical staining were separately and semi-quantitatively assessed by considering both the "percentage of positive tumor cells" (range 0-100\%), and the "intensity of staining" (range: $0-3^{+}$). Therefore we calculated a "score of positivity" by multiplying the value of the percentage of positive tumor cells for the value of staining intensity.

\section{Data management and statistical analysis}

Primary endpoint of this study was to evaluate the prognostic role of Hh pathway. Secondary endpoint was to evaluate the impact on outcome in those patients who received erlotinib.

Patients who were not reported as having died at the time of the analysis were censored at the date they were last known to be alive.

Progression-free survival (PFS) was defined as the interval between the start of treatment to disease progression or death, while overall survival (OS) was defined as the interval between histological diagnosis to death or last follow-up visit.

Survival distribution was estimated by the Kaplan-Meyer method. Significant differences in probability of surviving between the strata were evaluated by log-rank test. The association between categorical variables was estimated by Chi-square test.

The Cox multivariate proportional hazard regression model was used to evaluate the effects of the prognostic factors on PFS and OS.

A significant level of 0.05 was chosen to assess the statistical significance.

Statistical analysis was performed with MedCalc package (MedCalc ${ }^{\circ}$ v9.4.2.0).

\begin{tabular}{|l|l|}
\hline Patients Data & N (\%) \\
\hline Number of patients & $35(100)$ \\
\hline Platinum+pemetrexed & $18(51.4)$ \\
\hline
\end{tabular}


Citation: Berardi R, Santinelli A, Onofri A, Biscotti T, Ballatore Z, et al. (2014) Hedgehog (Hh) Signaling is a Predictor of Clinical Outcome for Advanced Non-Small Cell Lung Cancer (NSCLC). J Carcinog Mutagen 5: 175. doi:10.4172/2157-2518.1000175

Page 4 of 7

\begin{tabular}{|c|c|}
\hline Platinum+gemcitabina & $17(48.6)$ \\
\hline \multicolumn{2}{|l|}{ Clinical Benefit } \\
\hline Yes & $24(68.6)$ \\
\hline No & $9(26.5)$ \\
\hline Unknown & $2(5.9)$ \\
\hline \multicolumn{2}{|l|}{ Toxicity (GR3-4) } \\
\hline Anemia & $0(0)$ \\
\hline Neutropenia & $1(2.9)$ \\
\hline Thrombocytopenia & $0(0)$ \\
\hline \multicolumn{2}{|c|}{ Response to the first line } \\
\hline Complete response & $1(2,9)$ \\
\hline Partial response & 10(28.6) \\
\hline Stationarity of disease & $13(37.1)$ \\
\hline Progression of disease & $11(31.4)$ \\
\hline First line chemotherapy & $25(71.4)$ \\
\hline Pemetrexed & $1(4)$ \\
\hline Docetaxel & $0(0)$ \\
\hline Erlotinib & $24(96)$ \\
\hline Other & $0(0)$ \\
\hline \multicolumn{2}{|l|}{ Clinical Benefit } \\
\hline Yes & $6(24)$ \\
\hline No & $14(56)$ \\
\hline Unknown & $5(20)$ \\
\hline \multicolumn{2}{|l|}{ Toxicity (GR3-4) } \\
\hline Anemia & $1(0,9)$ \\
\hline Neutropenia & $1(0,9)$ \\
\hline Thrombocytopenia & $1(0,9)$ \\
\hline \multicolumn{2}{|c|}{ Response to the second line } \\
\hline Complete Response & $0(0)$ \\
\hline Partial Response & $5(20)$ \\
\hline Stationarity of Disease & $5(20)$ \\
\hline Progression of Disease & $14(60)$ \\
\hline
\end{tabular}

\section{Results}

\section{Sample characteristics}

Median age was 71 years (range $40-82$ ) and male/female ratio was $29 / 6(82.9 \% / 17.1 \%) ; 25(71.4 \%)$ patients were smokers or former smokers. Twenty patients (57.1\%) had ECOG 0 performance status, whilst 14 patients $(8 \%)$ had ECOG 1 performance status and only one had ECOG $\geq 2$. All the patients had advanced or metastatic disease. The most frequent stage at initial diagnosis was locally advanced or metastatic $(60 \%)$ and the most frequent sites of metastasis were lung and bone (Table 1).

According to the medical history, $14(40 \%)$ patients received radiotherapy, $15(42.9 \%)$ patients underwent surgery for primary tumor and among them, $7(46.7 \%)$ received subsequent adjuvant therapy (Table 1).

All patients received first line chemotherapy: 24 (68.6\%) experienced a clinical benefit and $23(65.7 \%)$ presented objective partial response or stable disease. One patient presented objective complete response (Table 2).

Nevertheless, 25 (71.4\%) patients received a second line chemotherapy due to progressive disease and 6 of them experienced a clinical benefit, while in 10 patients no evidence of objective partial response or stable disease was documented (Table 2).

During the chemotherapy treatments, less than $1 \%$ of patients reported grade 3-4 toxicity (according to CTC-CTAE v4.0).

In our series, median OS was 17.9 months (Figure 2). In all the patients, median PFS since first line chemotherapy was 7.4 months and median PFS since second line chemotherapy was 4.8 months (Figure 3).

According to PTCH intensity of expression, our study showed a significant correlation between overexpression of PTCH and OS $(\mathrm{p}=$ $0.047)$ and PTCH expression significantly increased in grading $1-2(\mathrm{p}$ $=0.041)$. In our study PTCH expression did not result associated with histotype $(\mathrm{p}=0.688)$.

\begin{tabular}{|l|l|l|l|l|}
\hline \multicolumn{6}{|l|}{ Relationship between PTCH expression and Survival } \\
\hline & 0 & 1 & 2 & 3 \\
\hline $\mathrm{N}^{\circ}$.of patients & 7 & 16 & 11 & 1 \\
\hline mOS & 148.197 & 179.016 & 21.377 & Not Reached \\
\hline $\mathrm{N}^{\circ}$.of patients & 7 & 16 & 11 & 1 \\
\hline First line PFS & 35.738 & 77.049 & 83.934 & Not Reached \\
\hline $\mathrm{N}^{\circ}$.of patients & 4 & 13 & 8 & \\
\hline Second Line PFS & 64.918 & 49.508 & 36.393 & \\
\hline
\end{tabular}

Table 2: First and second line chemotherapy characteristics and toxicity.

Table 3: $\mathrm{mOS}$ and PFS since first and second line of therapy in patients grouped according to PTCH intensity. 
Citation: Berardi R, Santinelli A, Onofri A, Biscotti T, Ballatore Z, et al. (2014) Hedgehog (Hh) Signaling is a Predictor of Clinical Outcome for Advanced Non-Small Cell Lung Cancer (NSCLC). J Carcinog Mutagen 5: 175. doi:10.4172/2157-2518.1000175

Page 5 of 7

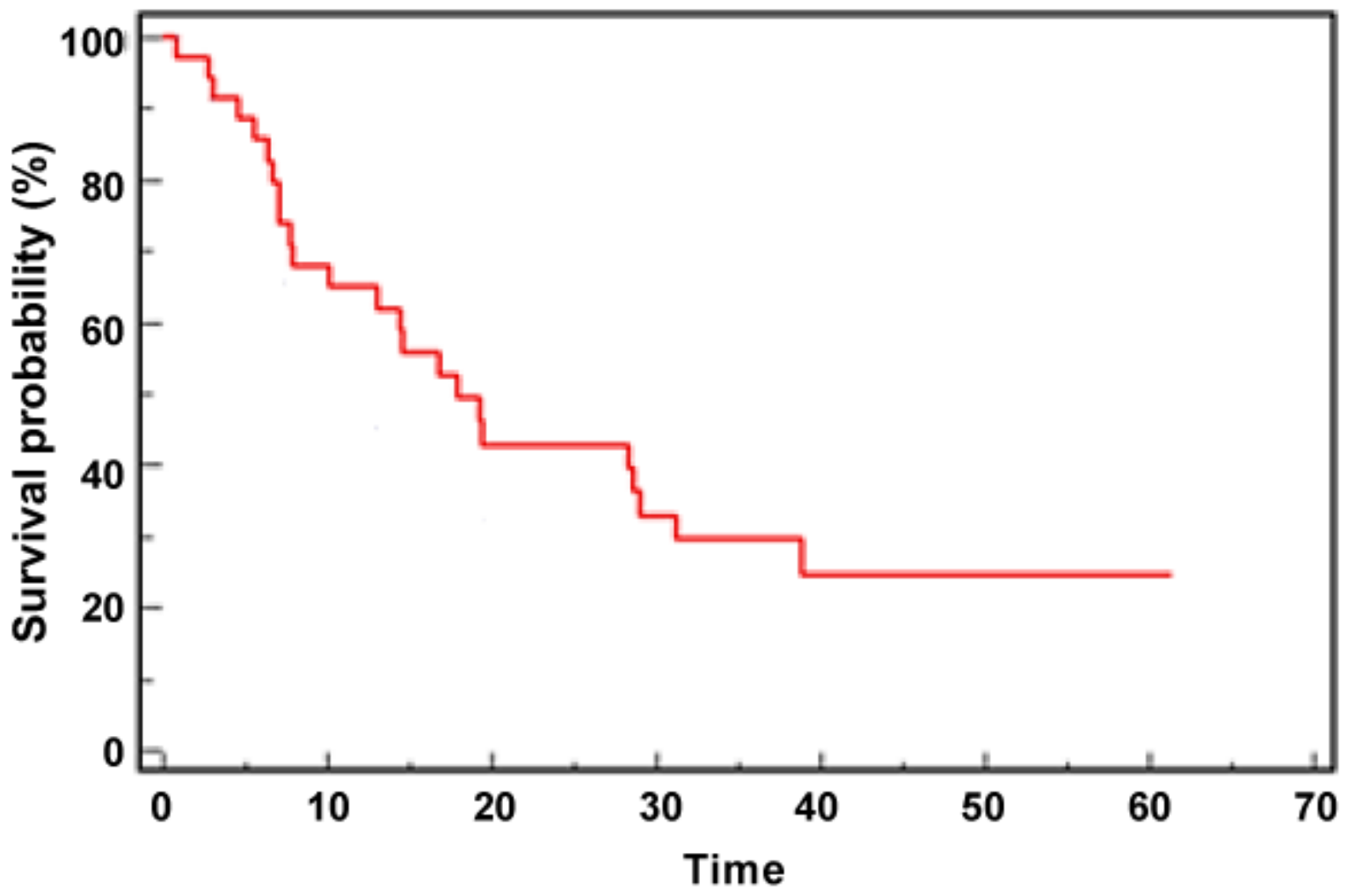

Figure 2: Overall Survival (OS) in all patients.

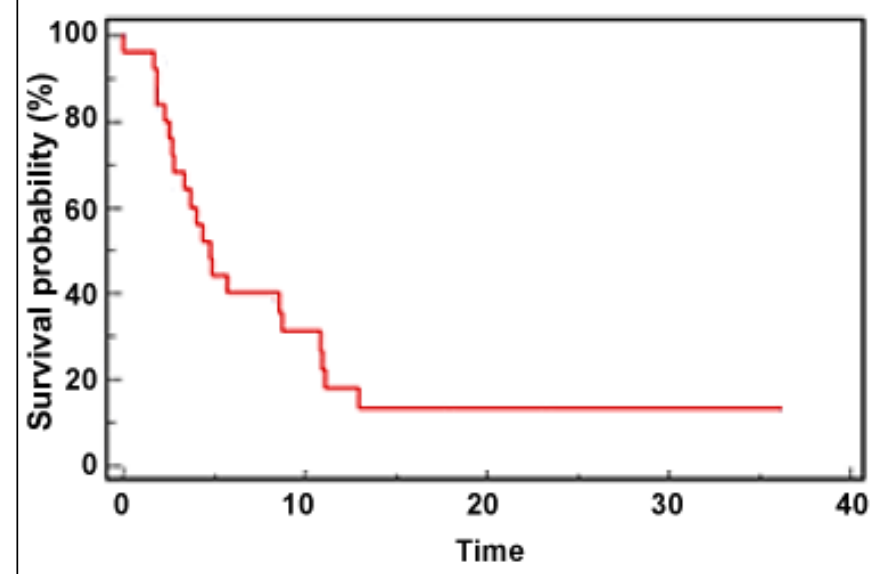

$3 \mathrm{~A}$

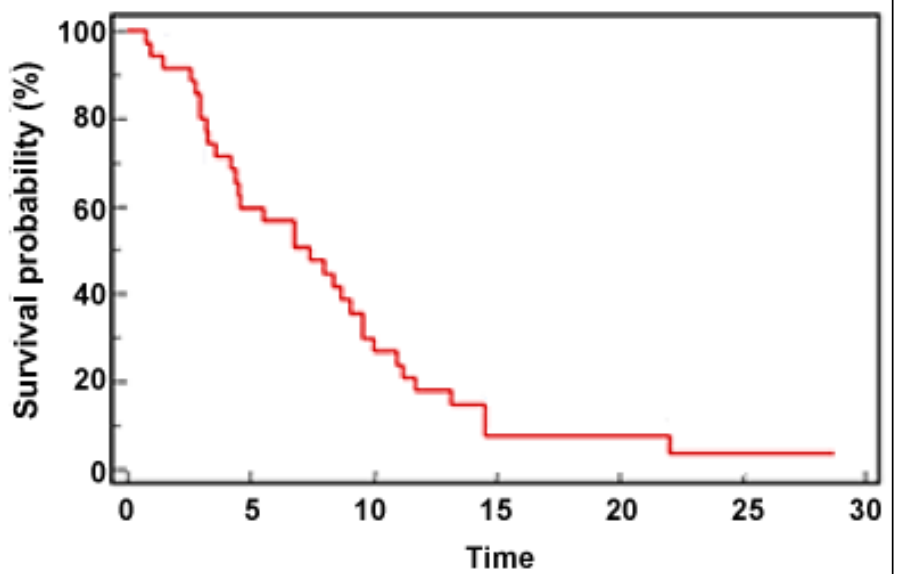

3B

Figure 3: A: Progression-Free Survival (PFS) at the first line of chemotherapy in all patients. B: Progression-Free Survival (PFS) at the second line of chemotherapy in patients treated with erlotinib.

Cytoplasmic and nuclear Gli1 was most frequently expressed in adenocarcinoma and to a significantly higher level than in squamous cell histotype $(\mathrm{p}=0.04)$. There was no correlation between the expression of cytoplasmic/nuclear Gli1 and the OS. However our results showed the evidence of a trend between PFS and expression of Glil. This trend reached the statistical significance considering PFS to the second-line therapy with erlotinib in patients with adenocarcinoma $(p=0.02)$. Furthermore the expression of GLI1 resulted significantly increased in adenocarcinoma histotype $(\mathrm{p}=$ $0.044)$.

Considering the Gli1 score, a significant correlation between the score and a better overall survival was showed (Table 4). 


\section{Relationship between GLI1 expression and Survival}

\begin{tabular}{|l|l|l|l|}
\hline & $\mathbf{1}$ & $\mathbf{2}$ & $\mathbf{3}$ \\
\hline No. of patients & 20 & 11 & 4 \\
\hline mOS & 18.04 & 10.11 & 05.24 \\
\hline No. of patients & 17 & 7 & 1 \\
\hline First line PFS & 87.705 & 4.623 & 2.332 \\
\hline
\end{tabular}

Table 4: $\mathrm{mOS}$ and PFS since first line chemotherapy in patients grouped according to the GLI1 intensity of expression. A trend between PFS and GLI1 expression is evident, but not statistical significance was reached.

We performed a multivariate analysis including sex, ECOG performance status, stage of disease at diagnosis and PTCH and Gli1 overexpression. Only sex, performance status, stage of disease and $\mathrm{PTCH}$ overexpression resulted independent prognostic factors.

\section{Discussion}

Signaling pathways responsible for embryogenesis play a critical role in the maintenance of stem cells in adult life and cellular responses to injury, as well as in cancer growth. Dysfunction of the $\mathrm{Hh}$ pathway during adult homeostasis leads to various events resulting in the development of neoplasia [20].

An important role of Hh pathway in SCLC has been also previously suggested $[12,14]$ and re-activation of $\mathrm{HH}$ pathway in adult lung, in response to injury, such as tobacco use, has been implicated in lung carcinogenesis $[16,21]$

Gialmanidis et al. demonstrated overexpression of Hh-signaling molecules in NSCLC compared to the adjacent normal lung parenchyma suggesting an involvement of $\mathrm{Hh}$ signaling in the pathogenesis of NSCLC [16]. Expression of SHH was detected in all the samples with a high expression of PTCH1 and GLI1, thus suggesting that $\mathrm{SHH}$ overexpression may be implicated in the activation of Hh signaling in NSCLC.

In order to evaluate the role of the Hh pathway in the clinical outcome of NSCLC patients, we primarily investigated the expression of PTCH1 and GLI1 by immunohistochemistry.

PTCH1 and GLI1 are two crucial target genes of the Hh pathway that seem to have been conserved throughout vertebrate evolution and their expression pattern reflects Hh-signaling activity [22].

Therefore, expression of both PTCH1 and GLI1 is considered an index of activation of the Hh pathway $[23,24]$.

Our results showed that cytoplasmic and nuclear Gli1 overexpression was more frequently expressed in adenocarcinoma and to a significantly higher level than in squamous cell histotype, but there is no correlation between the expression of cytoplasmic/nuclear Gli1 and OS.

According to literature data, this last result cannot be considered sufficient to preclude the role of $\mathrm{Hh}$ in increasing the aggressiveness of the disease being more invasive, giving lymph node metastases and implying a role of activated GLI1 in lung adenocarcinoma progression and metastases [16]. Furthermore, several studies highlighted the likely role of $\mathrm{Hh}$ in increasing the disease aggressiveness. Therefore GLI1 seems to correlate with a more aggressive disease. In addition, in the present study we also analyzed if GLI1 level might potential predict the efficacy of a second line treatment with erlotinib and we find a significant correlation between expression of Glil and PFS to the second-line therapy with erlotinib in patients affected by adenocarcinoma which showed a significant increased expression. Our findings provide a potential interesting starting point for new therapeutic strategies.

Our results showed no association between PTCH expression and histotype, nevertheless several studies reported that PTCH1 was overexpressed in NSCLC $[13,14]$ and its expression was significantly higher in lung squamous carcinoma cells [25]. Overexpression of PTCH1 seems to be implicated in other squamous neoplasms $[21,26,27]$ and loss of PTCH1 function seems to play a significant role in the oncogenic potential of human squamous carcinoma cell lines [28].

Besides in other malignancies PTCH1 expression was correlated with high grade and advanced stage tumors suggesting that $\mathrm{HH}$ signaling may differentially affect tumor biology depending on the tissue context $[29,30]$.

Furthermore Gialmanidis and colleagues showed that PTCH1 expression was higher in well differentiated NSCLC tumors and it was also inversely correlated with $\mathrm{pT}$ status in adenocarcinomas, indicating that increased levels of PTCH1 seem to correlate with parameters of early disease and favorable prognosis [16]. Our study shown that increasing score intensity PTCH expression corresponds to a statistically significant increase of the median survival $(p=0.047)$ and PTCH expression significantly increased in grade $1-2(\mathrm{p}=0.041)$. These findings are consistent with evidences of a recent report on gastric cancer patients with $\mathrm{Hh}$ pathway activation showing that PTCH1 expression was more frequently observed in well or moderately differentiated and low stage gastric cancer [31]. Based on these results PTCH1 seems to be a significant favorable indicator of better survival in oncology.

On the contrary, our results seemed to indicate a reverse trend in patients treated with erlotinib in second line: increasing intensity of PTCH expression correlates a decrease of PFS $(\mathrm{p}=0.03)$. This last finding is consistent with what Morgillo et al. [32] showed in vitro: PTCH mRNA levels resulted increased in TKI-resistant cell lines. That is of relevance because PTCH gene itself is a target gene for Gli1 transcriptional activity and its level could be an index of $\mathrm{HH}$ activation signaling in such cells. At the best of our knowledge, at present there are no other available literature data which could support this possible relationship, except of the cited Morgillo abstract. Therefore, further studies are needed to draw profitable conclusions.

Based on these controversial data, it is likely that further studies are needed to better define the true role of PTCH expression and lung cancer.

Furthermore, a recent article of Raza et al. investigated the possible correlation between Hh expression pathway and clinical outcomes in early-stage NSCLC patients and they did not find any significant correlation between the HHSP molecules and RFS or OS [15] concluding that, in early-stage NSCLC, HHSP protein expression does not offer prognostic value. Meanwhile, many authors suggested that HHSP can be activated in later stages of diseases in which HHSP activation has been shown to correlate with lower RFS and bad OS [33-37]. 


\section{Conclusion}

To the best of our knowledge this study is the first which investigated the association between $\mathrm{Hh}$ signaling pathway and prognosis and clinical outcomes in advanced stage of NSCLC. Despite the limitations given by the small number of samples examined in our study, we found the important prognostic role of Hh in lung cancer which is the most widespread cancer disease and yet the first cause of cancer death in the industrialized Countries.

Hh may represent a key disease pathway in progression therefore this pathway may hold promise for new therapeutic approaches.

\section{References}

1. Beckett WS (1993) Epidemiology and etiology of lung cancer. Clin Chest Med 14: 1-15.

2. World Health Organization (2011) Cancer institutional fact sheets.

3. Jemal A, Siegel R, Xu J, Ward E (2010) Cancer statistics, 2010. CA Cancer J Clin 60: 277-300.

4. Liu H, Gu D, Xie J (2011) Clinical implications of hedgehog signaling pathway inhibitors. Chin J Cancer 30: 13-26.

5. Kasper M, Regl G, Frischauf AM, Aberger F (2006) GLI transcription factors: mediators of oncogenic Hedgehog signalling. Eur J Cancer 42: 437-445.

6. Ingham PW, McMahon AP (2001) Hedgehog signaling in animal development: paradigms and principles. Genes Dev 15: 3059-3087.

7. Taipale J, Cooper MK, Maiti T, Beachy PA (2002) Patched acts catalytically to suppress the activity of Smoothened. Nature 418: 892-897.

8. Teglund S, Toftgård R (2010) Hedgehog beyond medulloblastoma and basal cell carcinoma. Biochim Biophys Acta 1805: 181-208.

9. Corbit KC, Aanstad P, Singla V, Norman AR, Stainier DY, et al. (2005) Vertebrate Smoothened functions at the primary cilium. Nature 437: 1018-1021.

10. Tostar U, Malm CJ, Meis-Kindblom JM, Kindblom LG, Toftgård R, et al. (2006) Deregulation of the hedgehog signalling pathway: a possible role for the PTCH and SUFU genes in human rhabdomyoma and rhabdomyosarcoma development. J Pathol 208: 17-25.

11. Bai LY, Chiu CF, Lin CW, Hsu NY, Lin CL, et al. (2008) Differential expression of Sonic hedgehog and Glil in hematological malignancies. Leukemia 22: 226-228.

12. Watkins DN, Berman DM, Burkholder SG, Wang B, Beachy PA, et al. (2003) Hedgehog signalling within airway epithelial progenitors and in small-cell lung cancer. Nature 422: 313-317.

13. Yuan Z, Goetz JA, Singh S, Ogden SK, Petty WJ, et al. (2007) Frequent requirement of hedgehog signaling in non-small cell lung carcinoma. Oncogene 26: 1046-1055.

14. Vestergaard J, Pedersen MW, Pedersen N, Ensinger C, Tümer Z, et al. (2006) Hedgehog signaling in small-cell lung cancer: frequent in vivo but a rare event in vitro. Lung Cancer 52: 281-290.

15. Raz G, Allen KE, Kingsley C, Cherni I, Arora S, et al. (2012) Hedgehog signaling pathway molecules and ALDH1A1 expression in early-stage non-small cell lung cancer. Lung Cancer 76: 191-196.

16. Gialmanidis IP, Bravou V, Amanetopoulou SG, Varakis J, Kourea H, et al. (2009) Overexpression of hedgehog pathway molecules and FOXM1 in non-small cell lung carcinomas. Lung Cancer 66: 64-74.

17. Edge SB, Byrd DR, Compton CC, Fritz AG, Greene FL et al. (2010). AJCC cancer staging manual (7th ed). Springer New York.

18. Edge SB, Compton CC (2010) The American Joint Committee on Cancer: the 7th edition of the AJCC cancer staging manual and the future of TNM. Ann Surg Oncol 17: 1471-1474.
19. Bogaerts J, Ford R, Sargent D, Schwartz LH, Rubinstein L, et al. (2009) Individual patient data analysis to assess modifications to the RECIST criteria. Eur J Cancer 45: 248-260.

20. Velcheti V, Govindan R (2007) Hedgehog signaling pathway and lung cancer. J Thorac Oncol 2: 7-10.

21. Lemjabbar-Alaoui H1, Dasari V, Sidhu SS, Mengistab A, Finkbeiner W, et al. (2006) Wnt and Hedgehog are critical mediators of cigarette smokeinduced lung cancer. PLoS One 1: e93.

22. van Tuyl M, Post M (2000) From fruitflies to mammals: mechanisms of signalling via the Sonic hedgehog pathway in lung development. Respir Res 1: 30-35.

23. Huang S, He J, Zhang X, Bian Y, Yang L, et al. (2006) Activation of the hedgehog pathway in human hepatocellular carcinomas. Carcinogenesis 27: 1334-1340

24. Ma X, Sheng T, Zhang Y, Zhang X, He J, et al. (2006) Hedgehog signaling is activated in subsets of esophageal cancers. Int J Cancer 118: 139-148.

25. Fujita E, Khoroku Y, Urase K, Tsukahara T, Momoi MY, et al. (1997) Involvement of Sonic hedgehog in the cell growth of LK-2 cells, human lung squamous carcinoma cells. Biochem Biophys Res Commun 238: 658-664.

26. Xuan YH, Jung HS, Choi YL, Shin YK, Kim HJ, et al. (2006) Enhanced expression of hedgehog signaling molecules in squamous cell carcinoma of uterine cervix and its precursor lesions. Mod Pathol 19: 1139-1147.

27. Yoshikawa R, Sasako M, Fujiwara Y (2008) Reply: molecular determinants of prognosis in oesophageal cancer patients treated with chemoradiation. Br J Cancer 99 : 1196.

28. Koike C, Mizutani T, Ito T, Shimizu Y, Yamamichi N, et al. (2002) Introduction of wild-type patched gene suppresses the oncogenic potential of human squamous cell carcinoma cell lines including A431. Oncogene 21: 2670-2678.

29. Ma X, Chen K, Huang S, Zhang X, Adegboyega PA, et al. (2005) Frequent activation of the hedgehog pathway in advanced gastric adenocarcinomas. Carcinogenesis 26: 1698-1705.

30. Feng YZ, Shiozawa T, Miyamoto T, Kashima H, Kurai M, et al. (2007) Overexpression of hedgehog signaling molecules and its involvement in the proliferation of endometrial carcinoma cells. Clin Cancer Res 13: 1389-1398.

31. Lee SJ, Do IG, Lee J, Kim KM, Jang J, et al. (2013) Gastric cancer (GC) patients with hedgehog pathway activation: PTCH1 and GLI2 as independent prognostic factors. Target Oncol 8: 271-280.

32. Morgillo F, Della Corte CM, G. Martini, Manzo A, Gambardella V et al. (2012). Role of the hedgehog pathway in mediating resistance to anti-egfr tyrosine kinase inhibitors in non small cell lung cancer. 37th ESMO congress, Vienna

33. Chen M, Hildebrandt MA, Clague J, Kamat AM, Picornell A, et al. (2010) Genetic variations in the sonic hedgehog pathway affect clinical outcomes in non-muscle-invasive bladder cancer. Cancer Prev Res (Phila) 3: 1235-1245

34. Dai J, Ai K, Du Y, Chen G (2011) Sonic hedgehog expression correlates with distant metastasis in pancreatic adenocarcinoma. Pancreas 40: 233-236.

35. Kim TJ, Lee JY, Hwang TK, Kang CS, Choi Yj (2011) Hedgehog signaling protein expression and its association with prognostic parameters in prostate cancer: a retrospective sturdy from the view point of new 2012 anatomic stage/prognostic group. Journal of surgical Oncology 104 : 472-479.

36. He HC, Chen JH, Chen XB, Qin GQ, Cai C, et al. (2012) Expression of hedgehog pathway components is associated with bladder cancer progression and clinical outcome. Pathol Oncol Res 18: 349-355.

37. Xu M, Li X, Liu T, Leng A, Zhang G (2012) Prognostic value of hedgehog signaling pathway in patients with colon cancer. Med Oncol 29: 1010-1016. 